\title{
Real Time Ultrasound in the Assessment of Intraocular Foreign Bodies
}

\author{
F. M. BRYDEN†, A. A. PYOTT, $\ddagger$ M. BAILEY, C. N. J. McGHEE§ \\ Glasgow
}

\begin{abstract}
Summary
The accurate detection and localisation of retained intraocular foreign bodies (IOFB) is important in the planning of subsequent surgical management. Using a porcine eye model, the authors have compared the relative detection rates of real time ultrasonography and plain roentgenograms for a variety of IOFBs. With an overall detection rate of $\mathbf{9 3 \%}$, ultrasonography appears to be a considerably more sensitive investigative tool than plain roentgenograms $(\mathbf{4 0} \%)$ for the imaging of IOFBs, particularly those that are non-metallic.
\end{abstract}

Ocular trauma remains the most common indication for enucleation in the young population and the presence or absence of an intraocular foreign body (IOFB) significantly affects the management of eyes that might be salvaged. ${ }^{1}$ With the advent of Computerised Tomography (CT) and Magnetic Resonance Imaging (MRI) very detailed images of the eye and orbital structures are now available. With these advances in technology one of the most readily available and inexpensive radio-

Table I

\begin{tabular}{lcc}
\hline Material & No of eyes & Range in size \\
\hline Glass & 30 & $7 \times 3 \times 1-2 \times 1 \times 1 \quad \mathrm{~mm}$ \\
Wood & 22 & $15 \times 2 \times 2-2 \times 1 \times 1 \quad \mathrm{~mm}$ \\
Plastic & 27 & $5 \times 5 \times 2-1.0 \mathrm{diam} \mathrm{mm}$ \\
Metal & 16 & $12 \times 1 \times 1-0.5$ diam mm \\
Graphite & 4 & $3 \times 1 \times 1-1.0$ diam mm \\
Control & 18 &
\end{tabular}

The composition and size of intraocular foreign bodies placed in 117 porcine eyes via an anterior scleral incision at the level of the pars plana. logical investigations is often overlooked or even considered 'yesterday's technology', however, orbital ultrasound remains a most useful, rapid and 'patient friendly' investigation in ocular disease. ${ }^{2}$

The most common IOFBs are composed of metal (from chisels, hammers, gunshot), wood (from circular saws, falls), glass (road traffic accidents, industrial accidents or assaults) and less commonly these foreign bodies may be plastic/perspex or graphite

Table II

\begin{tabular}{lcc}
\hline Foreign body & No of eyes & $\%$ identified \\
\hline Glass & 30 & $97 \%$ \\
Perspex & 27 & $96 \%$ \\
Wood & 22 & $91 \%$ \\
Graphite & 4 & $100 \%$ \\
Metal & 16 & $88 \%$ \\
\hline Total & 99 & $93 \%$
\end{tabular}

Identification of intraocular foreign bodies by real time ultrasound scanning using an ATL. 7.5 Megahertz real time scanning probe and Kitecko gel block.

From: †Department of Radiology, Southern General Hospital, Glasgow G51 4TF. $¥$ Department of Ophthalmology, Southern General Hospital, Glasgow G51 4TF. §The Tennent Institute, Western Infirmary, Glasgow G11 6NT.

Correspondence to: Dr C.N.J. McGhee, The Tennent Institute, Western Infirmary, 38 Church Street, Glasgow G11 6NT. 
Table III

\begin{tabular}{lcc}
\hline Foreign body & No of eyes & $\%$ identified \\
\hline Glass & 30 & $77 \%$ \\
Perspex & 27 & $15 \%$ \\
Wood & 22 & $9 \%$ \\
Graphite & 4 & $0 \%$ \\
Metal & 16 & $69 \%$ \\
\hline Total & 99 & $40 \%$ \\
\hline
\end{tabular}

Identification of intraocular foreign bodies by plain occipito-frontal and lateral X-rays.

(pencils). ${ }^{1,3,4}$ Due to hyphaemia, cataract or vitreous haemorrhage such IOFBs are often impossible to visualise by ophthalmoscopy and the clinician must rely upon radiographic techniques. In the investigation of any suspected penetrating ocular trauma, plain orbital X-rays (with exposure for soft tissues) are mandatory. Such X-rays will often reveal metallic IOFBs and any associated bony injury, however, they are less reliable in the
Table IV

\begin{tabular}{lcc}
\hline Investigation & False positives & False negatives \\
\hline X-Rays & $0 / 18(0 \%)$ & $59 / 99(60 \%)$ \\
Ultrasound & $2 / 18(11 \%)$ & $6 / 99(6 \%)$ \\
\hline
\end{tabular}

Comparison of the false positive and false negative rate. Eighteen eyes without intraocular foreign bodies which had been subjected to a small scleral stab incision were analysed to establish the false positive rates for plain roentgenography and ultrasound. These are compared to the false negative rates (missed IOFBs) for both techniques.

identification of retained wood or glass. The ability of plain roentgenography to detect an IOFB depends on the relative density of the IOFB compared with water. ${ }^{5}$

Obviously since many IOFBs are metallic Magnetic Resonance Imaging is not a suitable second line investigation and many experts would suggest that CT scan is the only second line investigation to consider. ${ }^{6-9}$ Unfortunately, due to the unavailability of CT facili-

\section{$\%$ IOFB's \\ DETECTED}
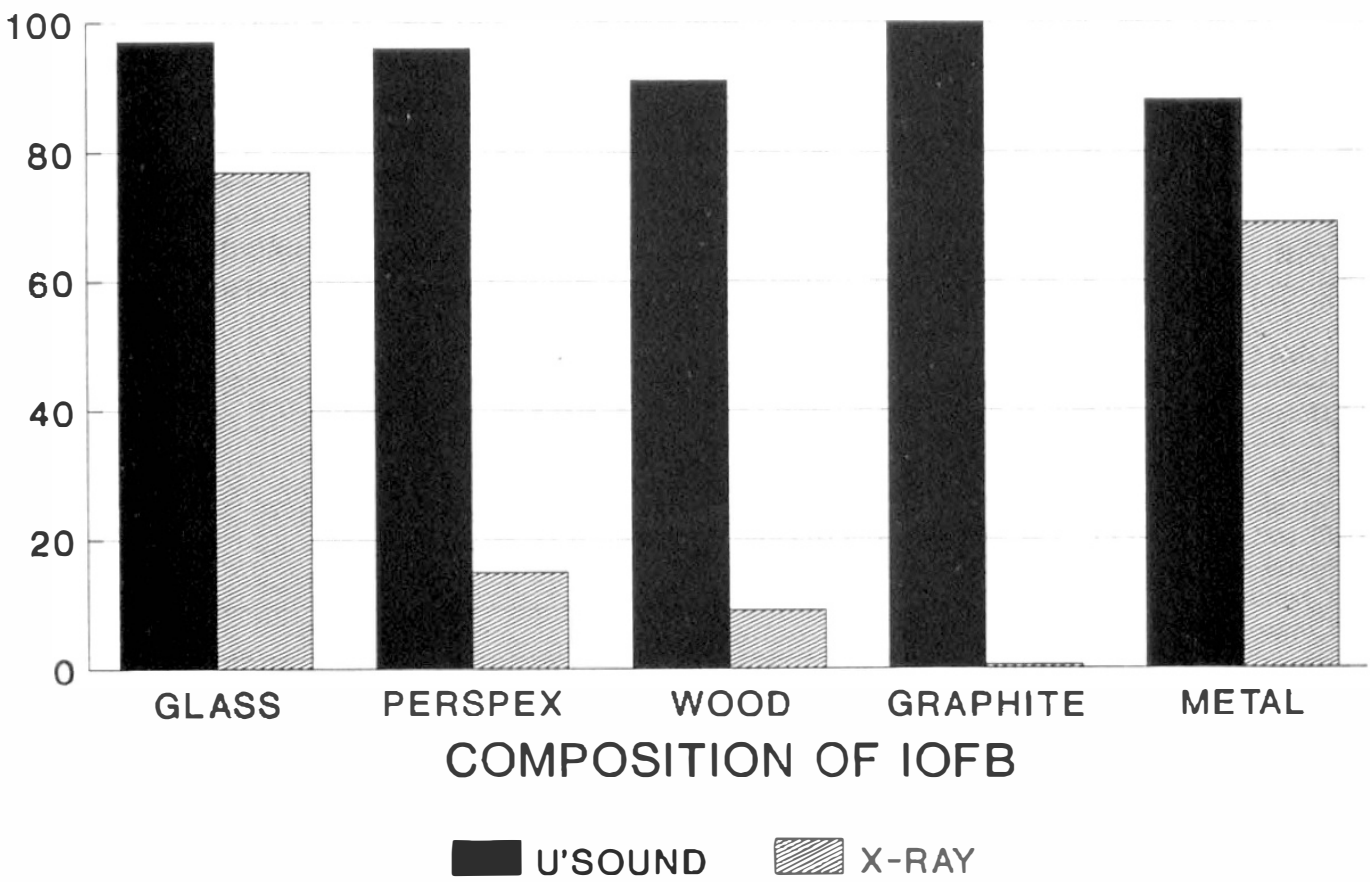

Fig. 1. Identification of intraocular foreign bodies by real time ultrasound compared to plain $X$-rays expressed as the percentage of IOFBs identified relative to the total number of IOFBs present. 


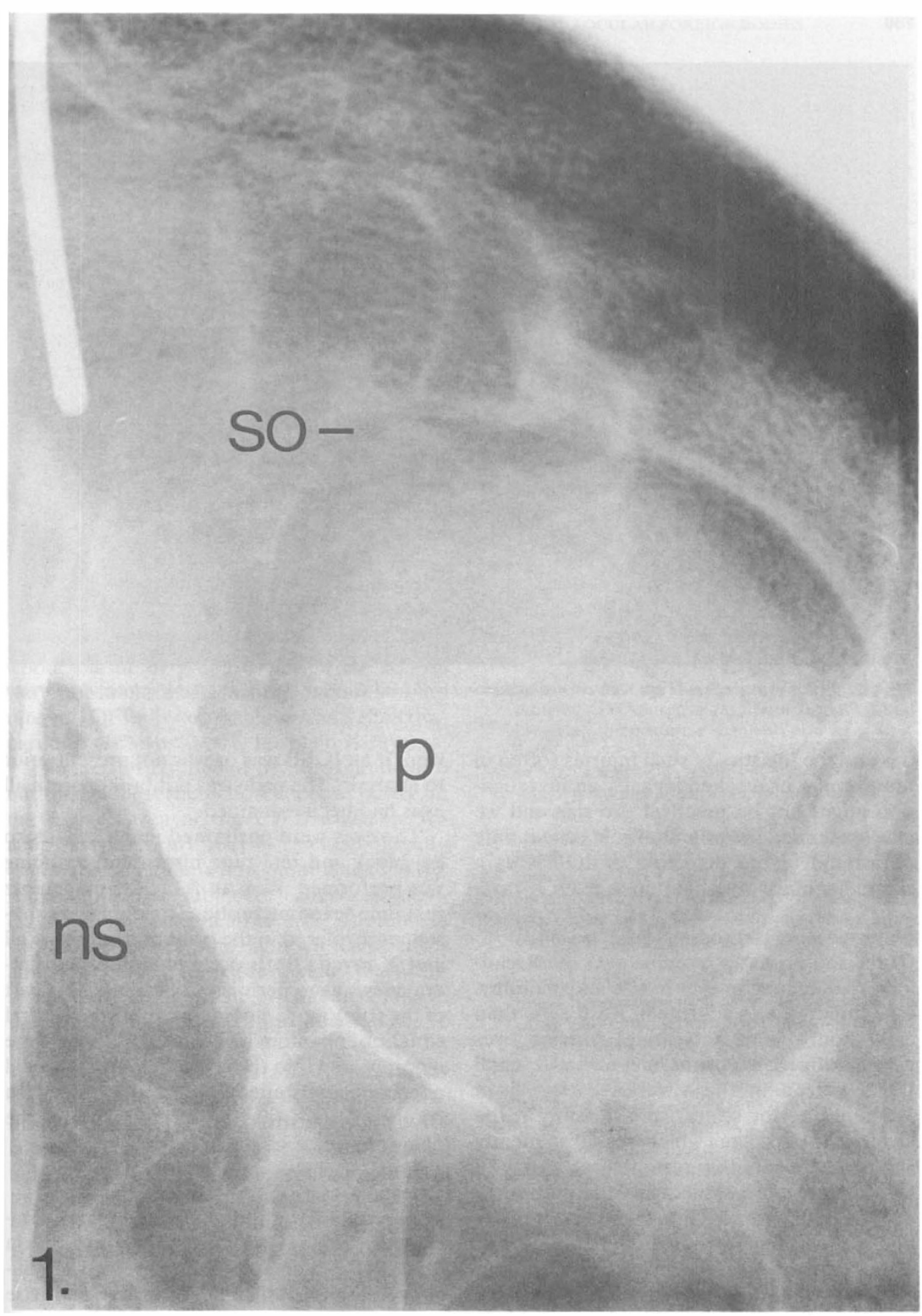

Fig. 2.1. An example of an intraocular foreign body in a porcine eye not detectable by plain $X$-ray but the same IOFB identified by real time ultrasonography: 1. Occipito-frontal $X$-ray; ns=nasal space, p=porcine eye, so=superior orbital margin. 


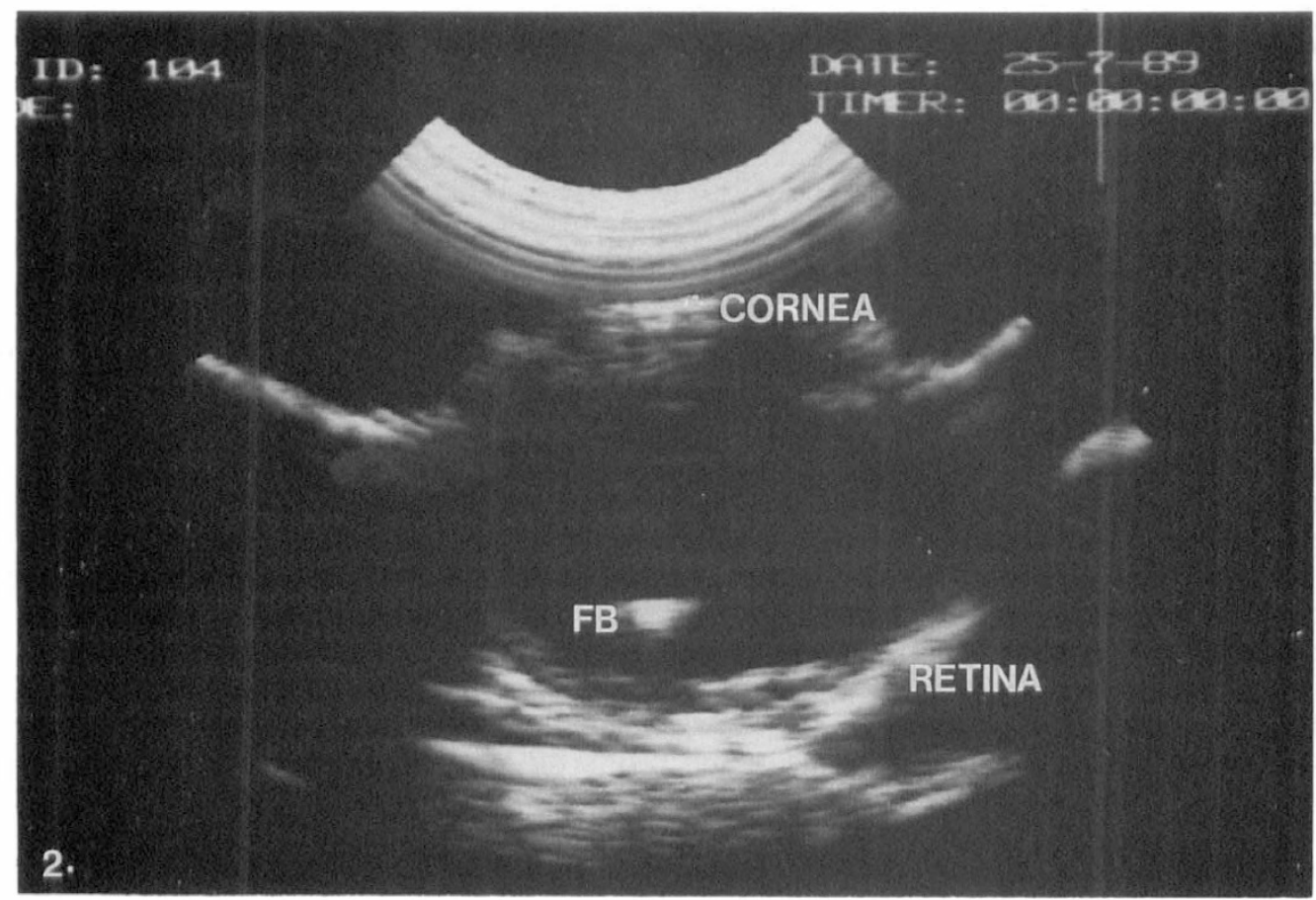

Fig. 2.2. Ultrasound of same eye with cornea and retina indicated showing clearly detectable intraocular foreign body $(F B)$.

ties and the logistics of such injuries (often in the evening or weekends) such an investigation might not be practical. To this end we have examined the potential role of real time ultrasound in the detection of IOFBs as a complementary investigation to plain X-rays.

\section{Materials and Methods}

Of the sub-primates, porcine eyes most readily resemble human eyes in size and anatomy. One hundred and seventeen fresh (less than eight hours from harvesting) porcine eyes were obtained; 99 of these eyes had a small IOFB placed in the vitreous cavity via a 3-5 $\mathrm{mm}$ anterior scleral stab incision at the level of the pars plana which was subsequently closed with a $5 / 0$ silk suture, the remaining 18 eyes underwent a similar incision and introduction of fine forceps into the vitreous cavity but without the introduction of an IOFB. These latter eyes served as the control group. The eyes were randomly allocated to receive one of the five types of foreign body (Table I) or to serve as controls and were identified by a code number. Thus the radiologist was una- ware if an IOFB was or was not present prior to analysis. The code was not broken until all eyes had been examined.

The eyes were positioned under a Kitecko gel block and real time ultrasound scanning was performed using an ATL. 7.5 megahertz real time scanning probe. The eyes were subsequently placed in the orbit of a human skull and X-rayed. Both occipito-frontal and lateral views were performed. To make this part of the study more closely resemble the clinical situation, phantom brain and soft tissue were incorporated into the skull, however, this did not significantly alter the detection rate when compared to X-rays performed without the phantom tissue.

\section{Results}

The results for orbital ultrasound are presented in Table II and those for Plain X-rays in Table III. The results are expressed graphically in Figure 1. The false positive and false negative rate is expressed in Table IV. When IOFB detection rates for ultrasound are compared to those for X-ray, it is noted in each 
group that more IOFBs are picked up by ultrasound: perspex $96 \%$ vs. $15 \%(\mathrm{p}<0.001)$, wood $91 \%$ vs. $9 \%(\mathrm{p}<0.001)$, graphite $100 \%$ vs. $0 \%(\mathrm{p}<0.07)$, glass $97 \%$ vs. $77 \%(\mathrm{p}=$ not significant) and metal $88 \%$ vs. $69 \%(\mathrm{p}=$ not significant). For technical reasons, one eye from the IOFB group and two eyes from the control group were excluded from analysis.

\section{Discussion}

Plain X-rays did identify many of the glass and metallic foreign bodies but almost $30 \%$ of the IOFBs in each group were missed. This failure to identify IOFBs did not relate to IOFB size in absolute terms and while the majority of the larger IOFBs were identified, it was not only the smallest which escaped detection. As might have been predicted, plain X-rays were much less good at picking up wood, plastic or graphite. The overall pick up rate for plain $\mathrm{X}$-rays was a surprisingly low $40 \%$.

In contrast ultrasound identified $88 \%$ of the metallic IOFBs and virtually all the glass IOFBs (97\%). Of the 53 eyes containing wood, plastic or graphite only three IOFBs were not identified. Thus the overall pick up rate of IOFBs by ultrasound was $93 \%$ compared to $40 \%(\mathrm{p}<0.001)$ for plain $X$-rays.

The six IOFBs missed by ultrasound were not the smallest (detection depends partly on the surface area of the IOFB available for acoustic reflection) ${ }^{10}$ but were either directly behind the crystalline lens or imbedded in the retina, both areas subject to 'acoustic shadowing' from the adjacent structures. There were two false positives from eighteen control eyes and it was felt that in these cases the ultrasound had picked up air or condensations in the vitreous at the site of introduction of the surgical instrument.

It appears from these data that orbital ultrasound is superior to plain X-rays in the identification of all IOFBs but particularly those not composed of metal or glass. With an overall detection rate of $93 \%$ these results might rival those of CT-scanning. However, unlike CTscanning, ultrasound is less good at identifying extraocular FBs in the posterior orbit. ${ }^{7}$ Although this ultrasound technique does not involve direct contact of the ultrasound probe with the eye, a gel block is placed on the closed lids, and while this does exert minimal compression upon the globe the technique would obviously not be suitable for large or unstable ocular wounds where there might be a risk of intraocular tissue prolapse. Nonetheless the authors believe that in cases of intraocular injury with small wounds and a suspected IOFB, the judicious use of orbital ultrasound may allow a rapid and accurate appraisal which will allow early management decisions to be made.

The authors wish to thank Ms S. McGlynn and Ms P. MacKay for their expert radiographic assistance.

\section{References}

${ }^{1}$ Bartkowski SB, Kurek M, Stypulkowska J, Krzystkowa K, Zapala J: Foreign bodies in the orbit: A review of 20 cases. J Maxillo-Facial Surg 1984, 12: 97-102.

${ }^{2}$ Byrne SF and Glaser JS: Orbital tissue differentiation with standardised echography. Ophthalmology 1983, 90: 1071-90.

${ }^{3}$ Brinton GS, Aaberg TM, Reeser FH, Topping TM, Abrams GW: Surgical results in ocular trauma involving the posterior segment. Am J Ophthalmol 1982, 93: 271-8.

${ }^{4}$ Cole MD, Clearkin L, Dabbs T, Smerdon D: The seat belt law and after. Br J Ophthalmol 1987, 71: $436-40$.

${ }^{5}$ De Lacey G, Evans R, Sandin B: Penetrating injuries: how easy is it to see glass (and plastic) on radiographs? Br J Radiol 1985, 58: 27-30.

${ }^{6}$ Etherington RJ and Hourihan MD: Localisation of intraocular and intraorbital foreign bodies using computed tomography. Clin Radiol 1989, 40: 610-14.

${ }^{7}$ Weisman RA, Savino PJ, Schut L, Schatz N: Computed tomography in penetrating wounds of the orbit with retained foreign bodies. Arch Otolaryngol 1983, 109: 265-8.

${ }^{8}$ Tate E and Cupples $\mathrm{H}$ : Detection of orbital foreign bodies with computed tomography: Current limits. Am J Roentgenol 1981, 137: 493-5.

${ }^{9}$ Zinreich J, Miller NR, Aguayo JB, Quinn C, Hadfield R, Rosenbaum AE: Computed tomographic three-dimensional localisation and compositional evaluation of introcular and orbital foreign bodies. Arch Ophthalmol 1986, 104: 1477-82.

${ }^{10}$ Awschalom L and Meyers S: Ultrasonography of vitreal foreign bodies in eyes obtained at autopsy. Arch Ophthalmol 1982, 100: 979-80. 